\title{
DRESS Syndrome-An Unusual Presentation with Pancytopenia after Salazopyrin Treatment
}

\author{
Adi Zoref-Lorenz ${ }^{1,2}$, Michal Slavin-Kish ${ }^{1,2}$, Itai Kalisky ${ }^{1,2}$, Elad Guber ${ }^{1,2}$, Eyal Robinson ${ }^{1,2}$, Gideon \\ Charach $^{1,2}$ and Mark Kheifets ${ }^{1,2 *}$ \\ ${ }^{1}$ Department of Internal Medicine B, Meir Medical Center, Kfar Saba, Israel
}

${ }^{2}$ Sackler School of Medicine, Tel Aviv University, Tel Aviv, Israel

*Corresponding author: Mark Kheifets, Department of Internal Medicine B, Meir Medical Center, Sackler Faculty of Medicine, Tel Aviv University, Tel Aviv, Israel.

To Cite This Article: Mark Kheifets, DRESS Syndrome-An Unusual Presentation with Pancytopenia after Salazopyrin Treatment. 2020 - 7(3). AJBSR.MS.ID.001144. DOI: 10.34297/AJBSR.2020.07.001144.

Received: 眥 January 28, 2020; Published: 䟱 February 14, 2020

\begin{abstract}
Background: Drug Reaction with Eosinophilia and Systemic Symptoms (DRESS) syndrome is a rare and potentially life-threatening adverse drug reaction. This syndrome is most frequently caused by various drugs such as allopurinol, Salazopyrin, antiepileptics (carbamazepine, lamotrigine, phenytoin, and phenobarbital), and antibiotics (sulfamethoxazole, dapsone, minocycline, and vancomycin). Treatment usually includes discontinuation of the offending drug and initiation of high-dose steroids with a prolonged taper.

Objective: To describe an unusual case of DRESS syndrome with pancytopenia during the time of initial presentation, following a late appearance of rash and eosinophilia.

Case Presentation: A 44-year-old woman was hospitalized in our internal medicine ward due to fever, cervical lymphadenopathy, pancytopenia and elevated liver enzymes one month after Salazopyrin therapy was initiated. A thorough investigation for malignant or viral etiology was performed and the lab results raised the suspicion of Hemophagocytic Lymphohistiocytosis (HLH). Subsequently, eosinophilia, atypical lymphocytes and a rash appeared 10 days after her initial presentation and DRESS syndrome was diagnosed.
\end{abstract}

Conclusion: DRESS syndrome is a unique, potentially life threatening, entity that can present in the form of fever and pancytopenia with delayed appearance of rash, eosinophilia and atypical lymphocytes. The recent use of drugs described as related to DRESS should raise the preliminary suspicion. In order to avoid complications, a high index of suspicion is warranted.

\section{Introduction}

Drug Reaction with Eosinophilia and Systemic Symptoms (DRESS) syndrome is a rare but potentially life-threatening adverse reaction that can result from exposure to a variety of medications. It most often develops after the use of sulfanilamide, allopurinol, phenytoin or other anticonvulsants. The syndrome is characterized by a late onset, usually occurring 2-6 weeks after exposure of the culprit drug, and a prolonged course. The clinical presentation varies greatly and includes a combination of fever, skin eruptions, lymphadenopathy, hematologic abnormalities (most often eosinophilia and atypical lymphocytes) and internal organ involvement [1]. As this presentation can mimic other conditions, mostly infec tion, diagnosis requires a high index of suspicion. We report a case of a 42-year-old woman who presented to our ward 4 weeks after initiation of Salazopyrin therapy, with fever, erythematous rash, pancytopenia, lymphadenopathy and hepatitis.

\section{Case Presentation}

A 44-year-old woman presented to the emergency department at our medical center after 4 days of weakness, fever $\left(39.5^{\circ} \mathrm{C}\right)$ and lymphadenopathy. No additional complaints were noted. A few months before her admission, she underwent medical investigation for sudden arthralgia. Salazopyrin therapy was initiated a month prior to the current hospitalization due to suspected spondyloarthropathy. Prior to hospitalization, she was treated with amoxicil- 
lin-clavulanic acid, clindamycin and corticosteroids by her family physician, with no improvement. On physical examination, the patient appeared ill with $1.5 \mathrm{~cm}$ tender cervical lymphadenopathy. Tonsils were not swollen and were clear of exudate.

Laboratory studies revealed a white cell count of $1500 / \mathrm{mm}^{3}$ with 830 neutrophils on the differential, hemoglobin $10.2 \mathrm{~g} / \mathrm{dl}$ and platelet count $90,000 / \mathrm{mm}^{3}$. A blood count taken 3 months prior to her admission was normal. Blood chemistry study showed C-reactive protein $3 \mathrm{mg} / \mathrm{dl}$. Liver enzymes were elevated: aminotransferase $227 \mathrm{u} / \mathrm{l}$, alanine transaminase $189 \mathrm{u} / \mathrm{l}$, gamma glutamyl transpeptidase $115 \mathrm{u} / \mathrm{l}$ and lactate dehydrogenase 2,212u/l. Chest radiograph was normal. The differential diagnosis upon initial presentation included drug reaction, lymphoproliferative disorder, infection or other inflammatory disease.

Upon hospitalization, Salazopyrin was discontinued. Urine and blood cultures were sterile, serologic tests for Epstein-Barr virus, cytomegalovirus, Hepatitis B and Hepatitis C were all negative. Serum ferritin was $1,141 \mathrm{mg} / \mathrm{dl}$ and fibrinogen and triglyceride levels were normal. Anti-nuclear and anti-RNP antibodies were positive. A total body CT revealed a normal-sized spleen with mild lymph- adenopathy in the axilla and mesentery. This was followed by a cervical ultrasound which demonstrated reactive lymph nodes. Bone marrow aspirate and biopsy were normal, with no evidence of aplasia, malignancy or hemophagocytosis. The high flares of fevers, pancytopenia and elevated ferritin raised the possibility of Hemophagocytic Lymphohistiocytosis (HLH).

Natural Killer (NK) cell activity level was 27\% (control = 67\%), and Soluble Interleukin 2 Receptor (sIL-2R) was 6,457u/ $\mathrm{mm}^{3}$ (normal $<2,000 \mathrm{u} / \mathrm{mm}^{3}$ ). In contrast to these results, the normal bone marrow examination, triglyceride levels and spontaneous drop in ferritin levels to $392 \mathrm{mg} / \mathrm{dl}$ made HLH a less probable diagnosis. The patient was started on high dose $(1 \mathrm{mg} / \mathrm{kg})$ corticosteroids which resulted in resolution of fever and improvement in cytopenia. She was subsequently discharged with a diagnosis of drug reaction. However, 48 hours after discharge, the patient was readmitted with temperature of $39.6^{\circ} \mathrm{C}$ and a new, erythema-like rash on her chest, back and legs, accompanied by facial edema and a pustular rash on her face. Episodes of high fever persisted, and 10 days after her initial presentation white blood count analysis revealed eosinophilia of $1200 / \mathrm{ml}^{3}$ with $15 \%$ atypical lymphocytes.
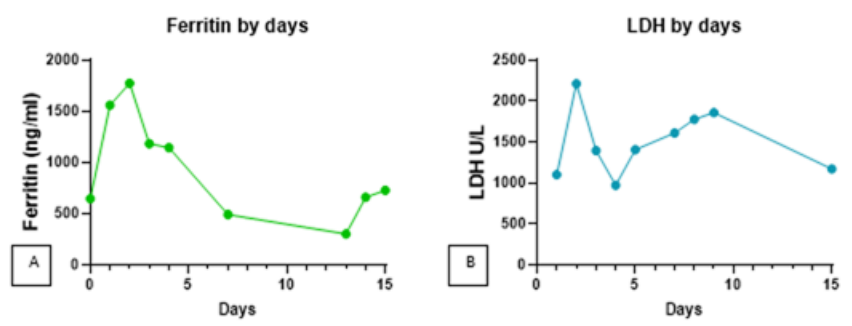

Blood count by days
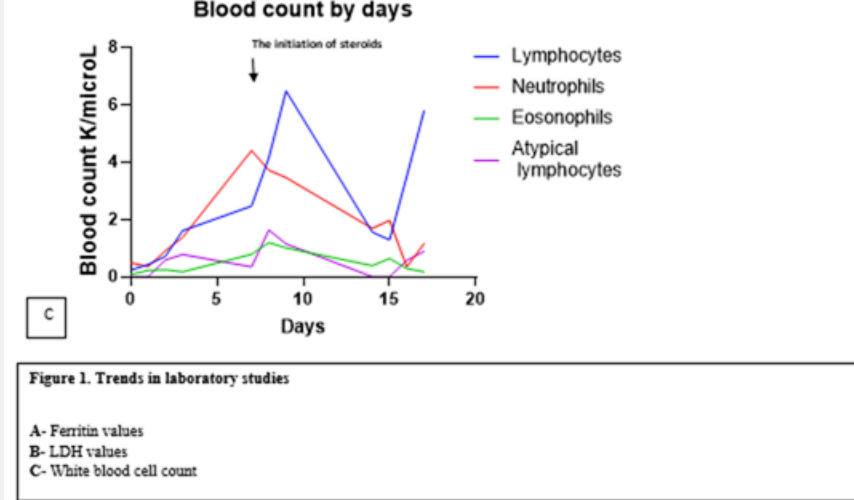

Figure 1: Trends in laboratory studies.

Hepatocellular liver enzymes were still high, at $>500 u / l$. Following these findings, despite the late erythematous eruption, DRESS syndrome was diagnosed. After high dose corticosteroid therapy was reinitiated, the patient reported marked clinical improvement-temperature returned to normal, the rash slowly disappeared, and lymphadenopathy resolved. Subsequent laboratory studies showed gradual improvement in ferritin, Lactate Dehydro- genase (LDH) and white blood count (Figure 1). The patient continued the high dose corticosteroid regimen for a full month, without tapering down, with complete normalization of clinical status and laboratory findings upon later office visits.

\section{Discussion}

DRESS syndrome is a potentially life-threatening syndrome that includes cutaneous eruptions, fever, hyper-eosinophilia and 
internal organ involvement (lungs, kidneys, liver) [2]. The estimated incidence of this syndrome ranges from 1 in 1,000 to 1 in 10,000 drug exposures [3]. It is considered a dermatological emergency with a mortality rate of approximately $10 \%$ [4]. The initial presentation of DRESS syndrome can often be misleading. The variability of initial features led to the development of the European Registry of Severe Cutaneous Adverse Reaction Criteria (RegiSCAR) scoring system, which is commonly used to aid diagnosis [5]

The patient described in this case first presented with high fever and lymphadenopathy four weeks after starting on Salazopyrin. Blood tests demonstrated pancytopenia and elevated liver enzymes, without hyper-eosinophilia or atypical lymphocytes. Only a week after initial presentation and 4 weeks after Salazopyrin was discontinued, a typical rash with marked eosinophilia and high, atypical lymphocyte count erupted. Despite this unusual presentation, a definitive diagnosis of DRESS syndrome was made according to the RegiSCAR scoring system: fever, lymphadenopathy, eosinophilia, elevated liver enzymes (as a marker for hepatitis and liver involvement), a rash that involves more than $50 \%$ of the body surface and facial edema. Surprisingly, the features presented by this patient also answer the 2004 revised diagnostic criteria for HLHfever, pancytopenia, elevated sIL-2R levels and reduced natural NK cell activity [6]. The overlap of DRESS and HLH were described in a few case reports in the literature [7-11]. It is still unclear whether HLH is a complication of DRESS or a situation that "mimics" this unique syndrome [12]. Although both syndromes reflect $\mathrm{T}$ cell hyper-activation, HLH classic treatment protocol involves the use of chemotherapy treatment [13].

The mainstay treatment of DRESS syndrome includes withdrawal of the suspected culprit drug and the use of systemic corticosteroids. High index of suspicion is warranted, since if administered in the acute stage of the disease, corticosteroids may improve the patient's long-term outcome [14]. Our patient's ferritin levels decreased dramatically following the initiation of steroid therapy, strengthening the diagnosis of DRESS syndrome and deferring the diagnosis of HLH.

\section{Conclusion}

In this report, we present a unique case of DRESS syndrome caused by salazopyrin and successfully treated by corticosteroid pulse therapy. Although DRESS and HLH syndromes both involve the activation of $\mathrm{T}$ cells and its subsequent complications, treatment strategies differ. Daily monitoring of ferritin, eosinophils, neutrophils, lymphocytes, TG and LDH will help establish the right diagnosis and prevent fatal complications. In cases of progressive pancytopenia, measurement of NK and sIL-2R levels is warranted.

\section{References}

1. Husain Z, Reddy BY, Schwartz RA (2013) DRESS syndrome: Part I. Clinical perspectives. J Am Acad Dermatol 68(5): 1-14.

2. Cacoub P, Musette P, Descamps V, Meyer O, Speirs C, et al. (2011) The DRESS Syndrome: A Literature Review. Am J Med 124(7): 588-597.

3. Albala FF, Auzerie V, Mahe E, Farinotti R, Stocco CD, et al. (2003) A 6-month prospective survey of cutaneous drug reactions in a hospital setting. Br J Dermatol 149(5): 1018-1022.

4. Chen YC, Chiu HC, Chu CY (2010) Drug reaction with eosinophilia and systemic symptoms: a retrospective study of 60 cases. Arch Dermatol 146(12): 1373-1379.

5. Kardaun SH, Sekula P, Allanore LV, Liss Y, Chu CY, et al. (2013) Drug reaction with eosinophilia and systemic symptoms (DRESS): an original multisystem adverse drug reaction. Results from the prospective RegiSCAR study. Br J Dermatol 169(5): 1071-1080.

6. Henter JI, Horne A, Arico M, Egeler RM, Filipovich AH, et al. (2007) HLH-2004: diagnostic and therapeutic guidelines for hemophagocytic lymphohistiocytosis. Pediatr Blood Cancer 48(2): 124-131.

7. Lakhoua G, Aouinti I, Sahnoun R, Kastalli S, Daghfous R, et al. (2016) A hemophagocytosis syndrome attributed to phenobarnital. Presse Med 45(3): 379-381.

8. Korbi M, Youssef M, Brahim HB, Chaabane A, Mohamed M, et al (2015) Allopurinol-induced DRESS complicated by hemophagocytic lymphohistiocytosis. J Ann Dermatol Venereol 142(12): 767-770.

9. Gauchan D, Shaaban H, Parikh N, Chang NL, Altheeb Z, et al. (2015) Severe hemophagocytic lymphohistiocytosis as a complication of drug induced hypersensitivity syndrome. Int Crit Illn Inj Sci 5(1): 60-61.

10. Picard M, Fernandez MI, Des Roches A, Bégin P, Paradis J, et al. (2013) Ceftazidime-induced drug reaction with eosinophilia and systemic symptoms (DRESS) complicated by hemophagocytic lymphohistiocytosis. Allergy Clin Immunol Pract 1(4): 409-412.

11. Komatsuda A, Okamoto Y, Hatakeyama T, Wakui H, Sawada K (2008) Sulfasalazine-induced hypersensitivity syndrome and hemophagocytic syndrome associated with reactivation of Epstein-Barr virus. Clin Rheumatol 27(3): 395-397.

12. Jordan MB, Allen CE, Greenberg J, Henry M, Hermiston ML, et al. (2019) Challenges in the diagnosis of hemophagocytic lymphohistiocytosis: Recommendations from the North American Consortium for Histiocytosis (NACHO). Pediatr Blood Cancer 66(11): 27929.

13. Risma KA, Marsh RA (2019) Hemophagocytic Lymphohistiocytosis: Clinical Presentations and Diagnosis. J Allergy Clin Immunol Pract 7(3): 824-832.

14. Shiohara T, Kano Y, Hirahara K, Aoyama Y (2017) Prediction and management of drug reaction with eosinophilia and systemic symptoms (DRESS) Expert Opinion on Drug Metabolism \& Toxicology 13(7): 701704. 